\title{
Enforced SOCS1 and SOCS3 expression attenuates Lck-mediated cellular transformation
}

\author{
JOHN C. COOPER ${ }^{1 *}$, MINGJIAN SHI $^{1,2^{*}}$, FU-YU CHUEH $^{1,3}$, \\ SRIVIDYA VENKITACHALAM ${ }^{3}$ and CHAO-LAN YU ${ }^{1,3}$ \\ ${ }^{1}$ Department of Molecular Physiology and Biophysics, Vanderbilt University School of Medicine, Nashville, TN 37221 , \\ USA; ${ }^{2}$ Department of Pharmacology, Wuhan University School of Medicine, Wuhan, P.R. China; ${ }^{3}$ Department of \\ Microbiology and Immunology, H.M. Bligh Cancer Research Laboratories, Chicago Medical School, \\ Rosalind Franklin University of Medicine and Science, North Chicago, IL 60064, USA
}

Received November 30, 2009; Accepted January 13, 2010

DOI: 10.3892/ijo_00000603

\begin{abstract}
Lck is an Src family protein tyrosine kinase with predominant $\mathrm{T}$ cell expression. Aberrant expression or activation of Lck kinase has been reported in both lymphoid and non-lymphoid malignancies. We showed previously that the signal transduction pathway involving Janus kinase (JAK) and signal transducer and activator of transcription (STAT) is constitutively activated and contributes to Lck-mediated oncogenesis. Under normal physiological conditions, active STAT proteins induce the expression of suppressor of cytokine signaling (SOCS) family proteins to inhibit further JAK/ STAT signaling. It is not fully understood whether and how SOCS-mediated negative feedback control is dysregulated in Lck-transformed cells. Here we report that two SOCS family members, SOCS1 and SOCS3, are not expressed in Lcktransformed LSTRA leukemia. While SOCS1 gene is silenced by DNA hypermethylation, loss of SOCS3 expression is through a mechanism independent of epigenetic silencing by DNA methylation. Furthermore, ectopic expression of SOCS1 or SOCS3 leads to reduced cell proliferation and increased apoptosis in Lck-transformed cells. This is consistent with the attenuation of Lck kinase activity by exogenous SOCS1 or SOCS3 expression. Downstream STAT5 activity is also inhibited as shown by reduced STAT5 tyrosine phosphorylation and in vitro DNA binding. All together, our data highlight the importance of silencing multiple SOCS genes in tumorigenesis and support the roles of SOCS1 and SOCS3 as tumor suppressors toward oncogenic Lck kinase.
\end{abstract}

Correspondence to: Dr Chao-Lan Yu, Department of Microbiology and Immunology, Rosalind Franklin University of Medicine and Science, 3333 Green Bay Road, North Chicago, IL 60064, USA E-mail: chaolan.yu@rosalindfranklin.edu

*Contributed equally

Key words: SOCS1, SOCS3, Lck, STAT5, BaF3

\section{Introduction}

Suppressor of cytokine signaling (SOCS) modulates Janus kinase-signal transducer and activator of transcription (JAKSTAT) signaling in a negative feedback manner (1). Among the eight SOCS family members, cytokine-inducible SH2containing protein (CIS), SOCS1 and SOCS3 are best characterized for their regulation and functions. Upon cytokine stimulation, active JAK and downstream STAT proteins induce the expression of STAT-target genes, including the SOCS genes. Cytokine-induced SOCS1 and SOCS3 proteins bind to JAK directly or the JAK-proximal sites on cytokine receptors to inhibit JAK kinase activity. CIS competes with STAT proteins in binding to cytokine receptors and blocks cytokine-induced STAT activation. All together, they contribute to the down-regulation of JAK-STAT signaling and the transient kinetics of JAK-STAT activation by cytokines and growth factors. The physiological importance of SOCS1 and SOCS3 is demonstrated by the lethal phenotypes observed in knockout mice (2-4). However, the lack of phenotype in CIS knockout mice suggests functional redundancy among SOCS family members.

SOCS1 and SOCS3 are both capable of inhibiting other non-receptor protein tyrosine kinases, such as focal adhesion kinase (FAK) (5) and breast tumor kinase (Brk) (6). It suggests that SOCS1 and SOCS3 may also target oncogenic protein tyrosine kinases and function as tumor suppressors. Indeed, exogenous SOCS3 negatively regulates cell mobility by inhibiting FAK in a hepatocellular carcinoma cell line (7). Similarly, enforced SOCS1 expression inhibits oncogenic fusion protein TEL-JAK2 kinase, which correlates with reduced tumorigenicity of $\mathrm{BaF} 3$ cells transformed by TELJAK2 (8). It should be noted, however, that not all oncogenic protein tyrosine kinases are subjected to inhibition by SOCS proteins. For example, SOCS1 cannot reverse cellular transformation induced by v-Src (8) and v-Abl (9). Therefore, tumorsuppressing activity of SOCS can be greatly affected depending on the oncoproteins and the intracellular environment of the tumor cells.

Constitutive activation of the JAK-STAT pathway is frequently associated with oncogenic protein tyrosine kinases 
and is reported in a wide variety of human cancers (10). A causal relationship between STAT activation and tumorigenesis has also been established in distinct tumor models. These findings raise the possibility that the negative feedback control involving SOCS proteins may be defective in these malignant cells. The observation that fibroblasts lacking SOCS1 are more susceptible to transformation supports this hypothesis (8). Inhibition of SOCS3 activity in human hepatocellular carcinoma cells also promotes cell migration that contribute to metastasis (7). Further evidence comes from high frequencies of SOCS gene silencing by DNA hypermethylation in human cancers. SOCS1 gene silencing has been reported in both lymphoid and non-lymphoid malignancies (11-19). On the other hand, SOCS3 gene silencing was observed in cholangiocarcinoma (20), head and neck cancer (21), and lung cancer (22). Consistent with potential functional overlaps among SOCS family members, hypermethylation of more than one SOCS genes was also reported in certain human cancers $(23,24)$.

Src-family non-receptor protein tyrosine kinases play key roles in tumor development and are important molecular targets in cancer therapy (25). Lck is a Src-family kinase essential for $\mathrm{T}$ cell development and activation $(26,27)$. In humans, aberrant Lck expression and kinase activity are implicated in both lymphoid and non-lymphoid malignancies $(28,29)$. In transgenic mice, Lck overexpression leads to thymic tumors (27). We showed previously that STAT5b is not only constitutively activated (30), but also contributes to cell proliferation and resistance to apoptosis in Lck-transformed cells (31). We also demonstrated the loss of CIS expression in Lck-transformed LSTRA leukemia cells through a mechanism independent of gene silencing by DNA hypermethylation (32). In this study, we further examine the regulation of SOCS1 and SOCS3 gene expression and determine their biological effects in Lck-transformed cells.

\section{Materials and methods}

Cell lines and culture conditions. Mouse T cell lines, LSTRA and EL4, and the human myeloma cell line U266 were maintained in RPMI supplemented with $5 \%$ fetal calf serum (FCS) and 5\% calf serum. Mouse pro-B cell line BaF3 was cultured in RPMI supplemented with 5\% FCS, 5\% calf serum, and $10 \%$ conditioned medium containing interleukin-3 (IL-3). For cytokine stimulation experiments, $\mathrm{BaF} 3$ cells were deprived of IL-3 for $16 \mathrm{~h}$ and then either left unstimulated or stimulated with $10 \mathrm{ng} / \mathrm{ml}$ of recombinant mouse IL-3 (R\&D Systems, Inc., Minneapolis, MN, USA) for $30 \mathrm{~min}$ or $1 \mathrm{~h}$. BaF3 cells transformed by the oncogenic Lck (Y505F) kinase was established and maintained as previously described (31).

Northern blot analysis. Total RNAs were isolated from cells using TRIzol (Invitrogen Inc., Carlsbad, CA, USA) and the manufacturer's protocol. Total RNA $(5 \mu \mathrm{g})$ for each sample was analyzed by Northern blotting essentially as described previously (33). Transcripts of SOCS1, SOCS3, Bcl-xL and GAPDH were detected using the corresponding [32P]-labeled full-length cDNAs.

$R T-P C R$ analysis. Exponentially growing LSTRA and U266 cells were adjusted to $1 \times 10^{7}$ cells per $\mathrm{ml}$ in $10 \mathrm{ml}$ of culture medium on day 1 . Cells were treated with $2 \mu \mathrm{g} / \mathrm{ml}$ of 5 ' azacytidine (Sigma, Inc., St. Louis, MO, USA) or equal volume of vehicle (50\% acetic acid) in two 24-h pulses on days 2 and 5 as described elsewhere (34). On day 8, cells were harvested. Total RNAs were extracted by TRIzol and then reverse-transcribed using M-MLV reverse transcriptase (Promega, Inc., Madison, WI, USA) into cDNAs. PCR was performed according to standard protocol using an annealing temperature of $65^{\circ} \mathrm{C}$ for all primer sets. Primers for SOCS1 are 5'-caggtggcagccgacaatgc-3' (forward) and 5'-ccgccgtcg gggctctg-3' (reverse). Primers for GAPDH are 5'-agctcactg gcatggcttccgt-3' (forward) and 5'-gcctgcttcaccaccttcttgatgt-3' (reverse). Amplified products were electrophoresed in a $4 \%$ metaphor agarose (Cambrex, Inc., East Rutherford, NJ, USA) gel and then visualized by ethidium bromide staining.

Construction and transfection of plasmids. The empty vector pEF was created by restriction digestion of pEF-FLAG-I/ mSOCS1 with XbaI from NEB (Beverly, MA, USA). The two $X b a \mathrm{I}$ recognition sites are located just outside the $5^{\prime}$ and 3 ' ends of cDNA insert. The vector $\mathrm{pEF}$ was separated from cDNA insert by agarose gel electrophoresis, purified, and then selfligated with T4 DNA ligase. Lck-transformed BaF3 cells were transiently transfected with $30 \mu \mathrm{g}$ of pEF, pEF-FLAG-I/ $\mathrm{mSOCS} 1$, or pEF-FLAG-I/mSOCS3 by electroporation in a single pulse using the Cell Porator (BRL Life Technologies, Inc., Rockville, MD, USA) set at $300 \mathrm{~V}, 800 \mu \mathrm{F}$, and low ohms as described previously (31).

Cell number and viability. Total numbers of Lck-transformed $\mathrm{BaF} 3$ cells were calculated $2 \mathrm{~h}$ after transfection and compared to cell numbers $24 \mathrm{~h}$ post-transfection. Cell viability was determined by incubation of a small fraction of cells in $0.1 \%$ trypan blue dye. Both viable and dead cells were counted and the percentage of dead cells was calculated 2 and 24 h posttransfection.

Flow cytometry. Detection of Annexin V incorporation by flow cytometry was performed essentially as previously described (31). Alexa Fluor 647-conjugated Annexin V was purchased from Molecular Probes, Inc. (Eugene, OR, USA). Analysis was performed using a FACS Calibur flow cytometer (Becton-Dickinson, Inc., San Jose, CA, USA).

DNA fragmentation analysis. DNA fragmentation analysis was performed $24 \mathrm{~h}$ after transfection essentially as noted previously (31). Exponentially growing Lck-transformed BaF3 cells were also subjected to fragmentation analysis as a nontransfected control.

Immunoprecipitation and immunoblotting. Preparation of whole cell lysates and immunoprecipitations were done essentially as described before (31). Anti-Lck, anti-Myc, and anti-STAT5b antibodies were purchased from Santa Cruz Biotechnology, Inc. (Santa Cruz, CA, USA). Anti-STAT5 and anti-FLAG monoclonal antibodies used for immunoblotting were from BD Transduction Laboratories (San Diego, CA, USA) and Sigma (St. Louis, MO, USA), respectively. The 4G10 monoclonal antibody used for immunoblotting was produced by the Molecular Recognition Shared Resources Center at Vanderbilt University (Nashville, TN, USA). 
A

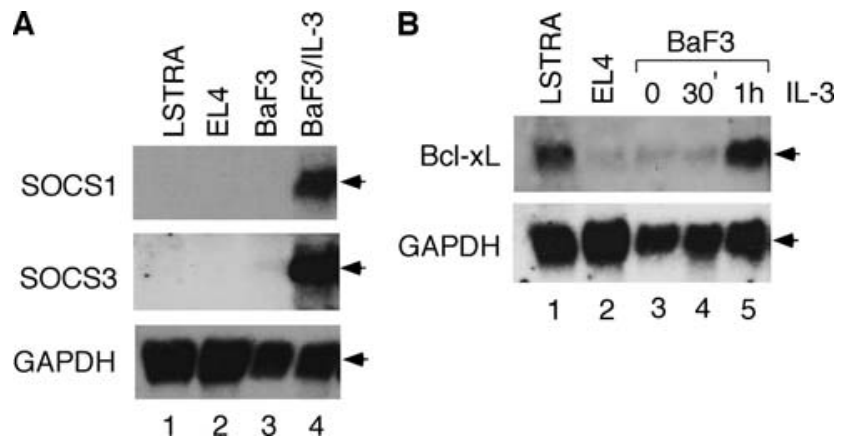

Figure 1. SOCS1 and SOCS3 are not expressed in LSTRA cells. (A) Total RNAs were isolated from exponentially growing LSTRA and EL4 cells as well as from BaF3 cells without or with 30 min of IL-3 stimulation. Northern blotting was performed with sequential probing using radiolabeled cDNA fragments of SOCS1, SOCS3 and GAPDH. (B) Total RNAs were isolated from exponentially growing LSTRA and EL4 cells as well as from BaF3 cells without (0) or with IL-3 stimulation for $30 \mathrm{~min}(30 ')$ and $1 \mathrm{~h}(1 \mathrm{~h})$. Total RNAs were analyzed by Northern blotting with a Bcl-xL-specific probe (upper panel). The membrane was then stripped and blotted with a GAPDHspecific probe as a normalization control (lower panel). Arrowheads denote correct positions of respective mRNAs.

Dilutions of different antibodies for immunoblotting and subsequent detection by the enhanced chemiluminescence system were done as recommended by the manufacturers.

In vitro kinase assays. A total of $1 \times 10^{6}$ cells were lysed in $200 \mu 1$ of lysis buffer (50 mM Tris pH 8.0, $150 \mathrm{mM} \mathrm{NaCl}$, $1 \mathrm{mM} \mathrm{Na} \mathrm{VO}_{4}, 10 \mathrm{mM} \mathrm{NaF}, 10 \mathrm{mM} \mathrm{Na}_{4} \mathrm{P}_{2} \mathrm{O}_{7}, 1 \mathrm{mM}$ EDTA, $1 \mathrm{mM}$ EGTA, 1\% Brij 35) with protease inhibitors and then used for Lck immunoprecipitation essentially as described previously (31). Immune complexes bound to beads were washed three times with $0.1 \%$ Brij 35 lysis buffer then washed with in vitro kinase reaction washing buffer $(150 \mathrm{mM} \mathrm{NaCl}$, $25 \mathrm{mM}$ HEPES pH 7.4, $\left.0.5 \mathrm{mM} \mathrm{Na}_{3} \mathrm{VO}_{4}\right)$. Substrates were phosphorylated with $1 \mathrm{mM}\left[\gamma^{-32} \mathrm{P}\right]-A T P(100-200 \mathrm{cpm} / \mathrm{pmol})$, along with $5 \mathrm{mM}$ HEPES pH 7.4, $0.1 \mathrm{mM} \mathrm{Na}_{3} \mathrm{VO}_{4}, 10 \mathrm{mM}$ $\mathrm{MnCl}_{2}, 5 \mathrm{mM}$ p-nitrophenyl phosphate. After incubation for $10 \mathrm{~min}$ at $30^{\circ} \mathrm{C}$, the immune complex was centrifuged briefly to remove unincorporated ${ }^{32} \mathrm{P}$. Reactions were terminated by the addition of SDS sample loading buffer and boiled for 5 min before being subjected to 7\% SDS-PAGE and subsequent transfer to nitrocellulose membrane. Intensity of ${ }^{32} \mathrm{P}$ incorporation was determined by autoradiography.

Electrophoretic mobility shift assay (EMSA). Preparation of nuclear extracts, electrophoretic mobility shift assays, and antibody supershift assays were all done essentially as described previously (30). The anti-STAT5b supershift antibody was purchased from Santa Cruz Biotechnology. The control rabbit antibody was from Southern Biotechnology Associates (Birmingham, AL, USA).

\section{Results}

Loss of SOCS1 and SOCS3 expression in LSTRA lymphoma. SOCS1 and SOCS3 down-regulate JAK-STAT signaling and inhibit distinct oncogenic protein tyrosine kinases. The absence of SOCS1 and SOCS3 gene expression has also
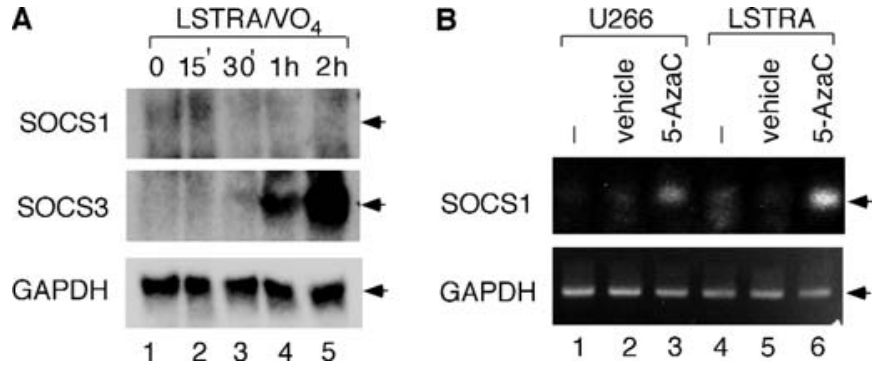

Figure 2. SOCS1, but not SOCS3, is silenced by DNA hypermethylation in LSTRA cells. (A) Exponentially growing LSTRA cells were treated with $100 \mu \mathrm{M}$ pervanadate $\left(\mathrm{VO}_{4}\right)$ for $15 \mathrm{~min}$ to $2 \mathrm{~h}$, and then analyzed by Northern blotting with sequential probing for SOCS1, SOCS3, and GAPDH transcripts. Arrowheads indicate correct positions of respective mRNAs. (B) LSTRA and U266 cells were either untreated (-) or treated with 5' aza-cytidine (5AzaC) or vehicle. Total RNAs were subjected to RT-PCR with SOCS1specific primers (upper panel). RT-PCR analysis with GAPDH-specific primers shows equivalent amount of total RNA (lower panel). Arrowheads indicate correct positions of $52 \mathrm{bp}$ (SOCS1) and $115 \mathrm{bp}$ (GAPDH) for regions amplified by sequence-specific primers.

been reported in several forms of solid tumors and blood malignancies. The mouse T lymphoma cell line LSTRA overexpresses active Lck kinase and is one of the bestcharacterized model systems for leukemogenesis (35). We showed previously that CIS, a known STAT5-target gene, was not expressed in LSTRA cells even though STAT5 was constitutively active $(30,32)$. It remains unknown, however, whether LSTRA exhibits similar dysregulation of SOCS1 and SOCS3 genes. Therefore, we examined SOCS1 and SOCS3 gene expression in LSTRA cells by Northern blot analysis (Fig. 1A). Using SOCS1 or SOCS3 full-length cDNA probe, we could not detect mRNA from either gene in LSTRA cells (lane 1, top and middle panels). Similarly, SOCS1 and SOCS3 transcripts were not detectable in EL4, a mouse $\mathrm{T}$ cell line expressing normal levels of Lck (lane 2, top and middle panels). As a positive control, IL-3 stimulation strongly activated SOCS1 and SOCS3 gene expression in BaF3 cells (compare lanes 3 and 4, top and middle panels). We confirmed equivalent loading of total RNAs by probing for GAPDH mRNAs (bottom panel). These results demonstrate that, in addition to CIS, SOCS 1 and SOCS3 gene expression is undetectable in LSTRA cells.

Similar to CIS, both SOCS1 and SOCS3 genes contain STAT5-binding sites within their promoter regions. Therefore, it was surprising that we were unable to detect the expression of SOCS1 and SOCS3 in LSTRA cells with constant STAT5 activation. To verify whether STAT5 is functional in activating other target genes in LSTRA cells, we examined the expression of Bcl-xL, an anti-apoptotic gene induced by active STAT5 (36). As shown in Fig. 1B, the levels of Bcl-xL transcripts were elevated in LSTRA cells (lane 1, upper panel). This was in sharp contrast to low Bcl-xL expression in EL4, which exhibit low levels of STAT5 activity (lane 2, upper panel). As a positive control, $\mathrm{Bcl}-\mathrm{xL}$ transcripts were induced in $\mathrm{BaF} 3$ cells following 1-h stimulation of IL-3 (lane 5, upper panel). Sequential blotting for GAPDH mRNAs confirmed equivalent loading of total RNAs (lower panel). From these observations, 
A

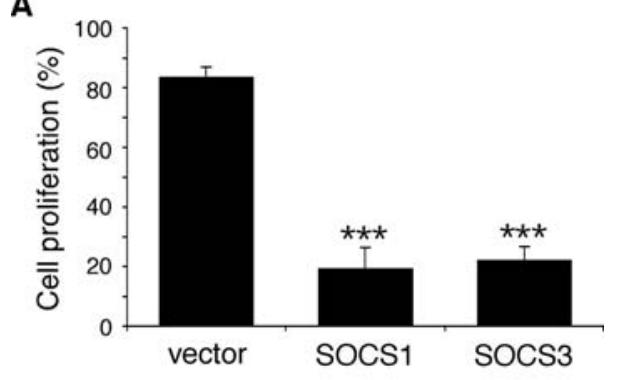

B

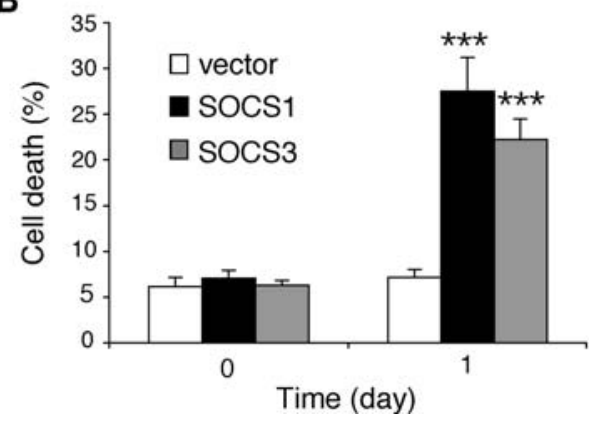

Figure 3. SOCS1 and SOCS3 inhibit cell proliferation and promote cell death in Lck-transformed $\mathrm{BaF} 3$ cells. Lck-transformed $\mathrm{BaF} 3$ cells were transfected with pEF (vector), pEF-FLAG-I/mSOCS1 (SOCS1), or pEFFLAG-I/mSOCS3 (SOCS3). At 2 and $24 \mathrm{~h}$ after transfection, a small fraction of Lck-transformed $\mathrm{BaF} 3$ cells were incubated with $0.1 \%$ trypan blue dye. After counting viable and dead cells, we calculated the percentage of viable cell increase (A) and the percentage of dead cells (B). Values represent means \pm SEM of six independent experiments. Statistical difference between SOCS1 or SOCS3 expressing cells and vector control cells in each group was determined by the Student's t-test $\left({ }^{* * *} \mathrm{P}<0.001\right)$.

we conclude that STAT5-target genes are differently regulated in LSTRA cells. While Bcl-xL expression is induced, three SOCS family members (CIS, SOCS1 and SOCS3) are not expressed in LSTRA leukemia.

SOCS1 gene silencing in LSTRA lymphoma. To determine whether the signaling pathways leading to SOCS1 and SOCS3 induction are functional, we performed pervanadate stimulation experiments in LSTRA cells. Pervanadate is a potent tyrosine phosphatase inhibitor that strongly activates tyrosine kinase signal transduction (37). We treated LSTRA cells with pervanadate for $15 \mathrm{~min}$ to $2 \mathrm{~h}$ and used Northern blot analysis to detect SOCS1 and SOCS3 gene expression. SOCS3 mRNA could be detected in LSTRA cells after stimulation with pervanadate for 1 and $2 \mathrm{~h}$ (middle panel, Fig. 2A). These results demonstrate that the machinery in activating SOCS3 gene expression is still intact in LSTRA cells. Reversal of DNA hypermethylation-mediated gene silencing depends on cell cycle progression and requires extended period of time (38). Therefore, the relatively fast SOCS3 induction by pervanadate also suggests that the absence of SOCS3 expression in LSTRA is independent of DNA hypermethylation.

In contrast to SOCS3, SOCS1 expression could not be detected during our time course of pervanadate treatment in LSTRA cells (top panel, Fig. 2A). It raised the possibility that SOCS1 gene might be silenced by DNA hypermethylation. To test this hypothesis, we used 5' aza-cytidine, a known DNA methyltransferase inhibitor, to reverse the effects
A

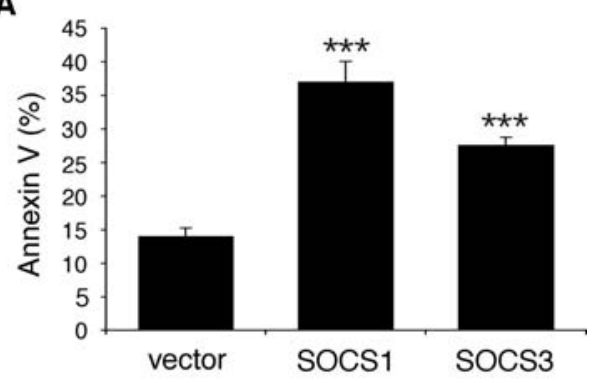

B

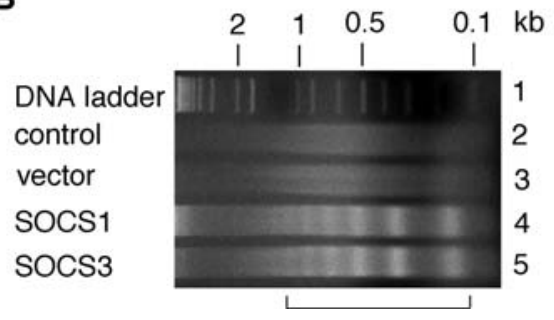

Figure 4. SOCS1 and SOCS3 enhance apoptosis in Lck-transformed BaF3 cells. Lck-transformed BaF3 cells were transfected with $\mathrm{pEF}$ (vector), pEFFLAG-I/mSOCS1 (SOCS1), or pEF-FLAG-I/mSOCS3 (SOCS3). (A) Cells were collected $24 \mathrm{~h}$ after transfection, stained with Alexa Fluor 647-conjugated Annexin V, and then analyzed by flow cytometry. Values represent means \pm SEM of three independent experiments. Statistical difference is determined when comparing either SOCS1 or SOCS3 expressing cells to vector-transfected cells $\left({ }^{* * *} \mathrm{P}<0.001\right)$. (B) Genomic DNAs were extracted from Lck-transformed BaF3 cells $24 \mathrm{~h}$ after transfected with pEF (vector), pEFFLAG-I/mSOCS1 (SOCS1), or pEF-FLAG-I/mSOCS3 (SOCS3). Genomic DNAs were also prepared from exponentially growing Lck-transformed $\mathrm{BaF} 3$ cells (control). DNA fragmentation was visualized after electrophoresis and ethidium bromide staining with a marker of $1 \mathrm{~kb}$ plus ladder (lane 1). The positions of $0.1,0.5,1$ and $2 \mathrm{~kb}$ are indicated on top. The bracket in the bottom denotes the lower molecular weight DNA fragments.

from DNA hypermethylation. As shown by RT-PCR analysis (Fig. 2B), SOCS1 gene expression could be reactivated by 5 ' azacytidine treatment, but not in untreated or vehicletreated LSTRA cells (lanes 4-6, top panel). We also examined the effects of $5^{\prime}$ azacytidine treatment on SOCS1 gene expression in U266 cells, a multiple myeloma cell line known to exhibit SOCS1 gene silencing by DNA hypermethylation (12). As expected, SOCS1 expression was induced in U266 cells following 5 ' azacytidine treatment, but not in untreated or vehicle-treated conditions (lanes 1-3, top panel). GAPDH detection in both Northern blot analysis and RT-PCR analysis confirmed equal loading of samples (bottom panels). These results point to the existence of two distinct mechanisms underlying the loss of SOCS1 and SOCS3 gene expression in LSTRA cells.

Inhibition of cell proliferation by exogenous SOCS1 and SOCS3. SOCS1 and SOCS3 have been shown to exert tumorsuppressing activity in certain tumor models (5-8). The observation that both SOCS1 and SOCS3 are not expressed in LSTRA leukemia cells suggests that SOCS1 and SOCS3 may also function as tumor suppressors in Lck-mediated oncogenesis. Because of the extreme difficulty in transfecting LSTRA cells, we examined another Lck-transformed cell line previously established in our laboratory (31). Mutation of the negative regulatory tyrosine 505 to phenylalanine in Lck 
A
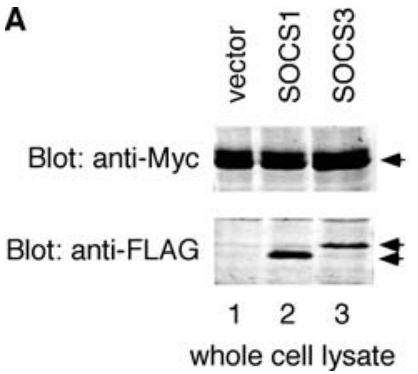

B
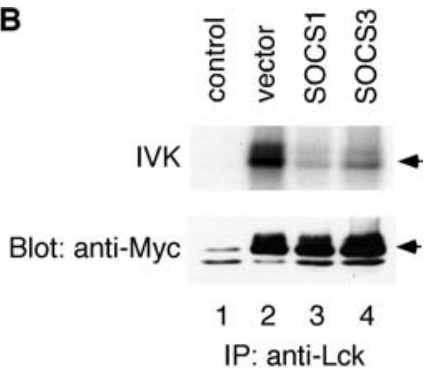

Figure 5. Lck kinase activity is inhibited in Lck-transformed BaF3 cells expressing SOCS1 or SOCS3. Lck-transformed BaF3 cells were transfected with pEF (vector), pEF-FLAG-I/mSOCS1 (SOCS1), or pEF-FLAG-I/ mSOCS3 (SOCS3). (A) A small aliquot of normalized whole cell lysates were analyzed by immunoblotting with anti-Myc (upper panel) and antiFLAG (lower panel) antibodies to determine the expression levels of exogenous Lck and SOCS proteins, respectively. (B) Proteins in the remaining lysates were immunoprecipitated with anti-Lck antibody and subjected to in vitro kinase assay (IVK). Anti-Lck immunoprecipitates were subjected to SDS-PAGE and transferred to membrane, followed by autoradiography (upper panel) and immunoblotting with anti-Myc antibody (lower panel). Parental BaF3 cells not expressing Lck (Y505F) serve as a negative control (lane 1). The arrowheads indicate the correct positions of Myc-tagged Lck (Y505F), FLAG-tagged SOCS1, or FLAG-tagged SOCS3.

results in a constitutively active kinase (39). Prolonged expression of Lck (Y505F) in IL-3-dependent BaF3 cells leads to IL-3-independent growth and resistance to apoptosis (31).

To examine the effects of enforced SOCS1 and SOCS3 expression in Lck-transformed $\mathrm{BaF} 3$ cells, we transiently transfected the cells with plasmids containing full-length cDNA for either gene with a FLAG epitope tag. There was a significant decrease in cell proliferation $24 \mathrm{~h}$ after transfection of either SOCS1 or SOCS3 in comparison to vector-transfected cells (Fig. 3A). SOCS1 and SOCS3 expression also led to a significant increase in cell death over this same time period (Fig. 3B). These results suggest that enforced SOCS1 or SOCS3 expression could reverse the malignant phenotypes in Lck-transformed BaF3 cells. Similar to Fig. 5A, the presence of SOCS1 and SOCS3 in transfected cells was confirmed by Western blot analysis using anti-FLAG antibody (data not shown).

Elevated apoptosis induced by exogenous SOCS1 and SOCS3. IL-3-dependent BaF3 cells undergo programmed cell death or apoptosis in the absence of the cytokine (40). To determine whether the increased cell death of Lck-transformed cells by exogenous SOCS1 and SOCS3 (Fig. 3B) is due to apoptosis, we performed Annexin $\mathrm{V}$ analysis and DNA fragmentation assay. Annexin V conjugates with phosphotidyl serines that have translocated to the outer leaflet of the plasma membrane, which is an event indicative of apoptotic cells (41). As shown in Fig. 4A, the percentage of Annexin V-positive cells was significantly higher in SOCS1- and SOCS3-transfected cells as compared to vector-transfected cells. Apoptotic cells also exhibit characteristic nuclear breakdown and chromatin shearing at regular intervals along nucleosomes that gives a pronounced laddering effect upon ethidium bromide staining (42). Consistent with elevated Annexin V staining, enforced

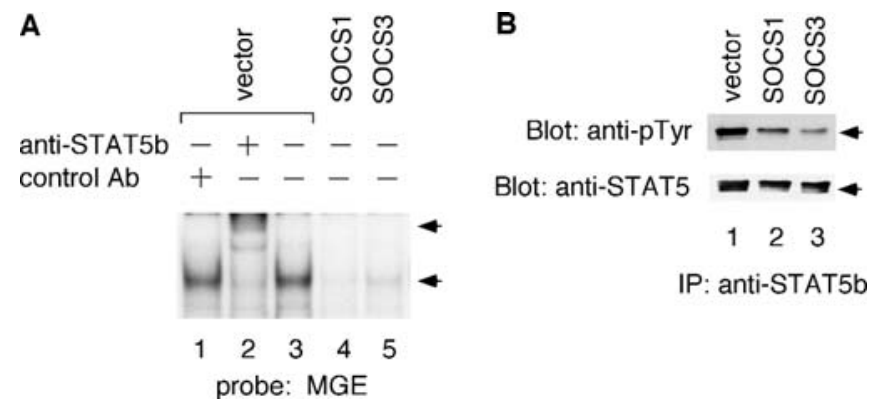

Figure 6. Exogenous SOCS1 and SOCS3 reduce STAT5b activity in Lcktransformed $\mathrm{BaF} 3$ cells. Lck-transformed $\mathrm{BaF} 3$ cells were transfected with pEF (vector), pEF-FLAG-I/mSOCS1 (SOCS1), or pEF-FLAG-I/mSOCS3 (SOCS3). (A) Nuclear extracts each containing $2 \mu \mathrm{g}$ of total proteins were subjected to EMSA with a ${ }^{32} \mathrm{P}$-labeled mammary gland element (MGE) that contains consensus STAT5-binding site. The lower arrowhead indicates the position of the STAT5b-DNA complex. Nuclear extract from vectortransfected cells was also subjected to antibody supershift with either antiSTAT5b antibody (lane 2) or control rabbit IgG antibody (lane 1). The upper arrowhead denotes the supershifted STAT5b-DNA complex. (B) Normalized whole cell lysates were subjected to immunoprecipitation using anti-STAT5b antibody. Proteins in the immunoprecipitates were resolved by SDS-PAGE followed by anti-phosphotyrosine immunoblotting (upper panel). The blot was stripped and then reprobed with anti-STAT5 antibody (lower panel). The arrowheads denote the correct positions of endogenous STAT5b.

SOCS1 and SOCS3 expression resulted in significant DNA fragmentation (Fig. 4B, lanes 4 and 5). As negative controls, no laddered shearing effect could be detected in non-transfected or vector-transfected cells (Fig. 4B, lanes 2 and 3). These results clearly show that ectopic SOCS1 or SOCS3 expression induces apoptosis in Lck-transformed BaF3 cells. Similar to Fig. 5A, the presence of SOCS1 and SOCS3 was confirmed by Western blot analysis using anti-FLAG antibody (data not shown).

Inhibition of Lck kinase activity by exogenous SOCS1 and SOCS3. Both SOCS1 and SOCS3 have been shown to inhibit JAK and several other protein tyrosine kinase activity. We hypothesize that SOCS1 and SOCS3 block Lck-mediated oncogenesis by inhibiting Lck kinase activity in Lck-transformed $\mathrm{BaF} 3$ cells. To evaluate Lck kinase activity, we performed in vitro kinase assay to measure Lck autophosphorylation in Lck-transformed BaF3 cells (Fig. 5B, upper panel). Consistent with its constitutively active nature, Lck (Y505F) prepared from vector-transfected Lck-transformed $\mathrm{BaF} 3$ cells showed high levels of ${ }^{32} \mathrm{P}$ incorporation due to autophosphorylation (lane 2). In contrast, Lck autophosphorylation was greatly reduced in cells expressing exogenous SOCS1 or SOCS3 (lanes 3 and 4). As a negative control, no phosphorylation could be detected in parental BaF3 cells lacking Lck expression (lane 1). The reduction of Lck kinase activity was not due to the amount of Lck proteins in the immunoprecipitates (Fig. 5B, lower panel, lanes 2-4) or in the whole cell lysates (Fig. 5A, upper panel). Expression of exogenous SOCS1 and SOCS3 was also confirmed by anti-FLAG immunoblotting (Fig. 5A, lower panel, lanes 2 and 3). We conclude from these experiments that Lck kinase activity is attenuated in cells expressing SOCS1 or SOCS3. 
Inhibition of STAT5 activity by exogenous SOCS1 and SOCS3. We showed previously that STAT5b plays a critical role in Lck-mediated oncogenesis (31). To determine whether expression of SOCS1 or SOCS3 affected STAT5b activity in Lck-transformed cells, we first examined the DNA binding activity of STAT5b. Similar to LSTRA cells, Lck-transformed $\mathrm{BaF} 3$ nuclear extracts exhibited elevated DNA binding activity to a consensus STAT5-binding sequence in vitro (Fig. 6A, lane 3). The identity of STAT5b binding to the probe was confirmed by supershifting with anti-STAT5b antibody, but not control IgG (Fig. 6A, lanes 1 and 2). As compared to the vector control, STAT5b DNA-binding activity was greatly reduced in cells expressing either SOCS1 or SOCS3 (Fig. 6A, lanes 4 and 5). It demonstrates that enforced SOCS1 or SOCS3 expression can suppress STAT5b DNA binding activity in Lck-transformed BaF3 cells.

Tyrosine phosphorylation is key to STAT5b function through dimerization, nuclear translocation, and DNA binding (43). We, therefore, further examined the levels of STAT5b phosphotyrosine in Lck-transformed BaF3 cells expressing SOCS1 or SOCS3 (Fig. 6B). Consistent with elevated DNAbinding activity, STAT5b immunoprecipitates showed high levels of tyrosine phosphorylation in vector-transfected cells (lane 1, upper panel). Similarly, STAT5b tyrosine phosphorylation was greatly reduced in cells expressing SOCS1 or SOCS3 (lanes 2 and 3, upper panel). Stripping and reblotting with anti-STAT5 antibody detected equivalent amounts of STAT5b in all immunoprecipitates (lower panel). These results illustrate that expression of SOCS1 or SOCS3 in Lck-transformed $\mathrm{BaF} 3$ cells also reduces STAT5b activity, which is downstream of Lck (Y505F). Altogether, we conclude that introduction of exogenous SOCS1 or SOCS3 can reverse Lck-mediated transformation in $\mathrm{BaF} 3$ cells by inactivating Lck and the downstream transcription factor STAT5b.

\section{Discussion}

SOCS1 and SOCS3 are closely related to each other both structurally and functionally. They can be induced by an overlapping set of cytokines, suggesting functional redundancy and potential compensation between SOCS proteins. Among eight SOCS family members, SOCS1 gene is the one most frequently silenced in human cancers. It is not known, however, whether SOCS3 or other SOCS proteins may compensate for the loss of SOCS1 expression in tumor cells. We demonstrate here that, in addition to CIS, both SOCS1 and SOCS3 are not expressed in Lck-transformed LSTRA leukemia cells. This novel finding suggests that loss of multiple SOCS gene expression may be necessary to overcome the functional overlap of SOCS proteins in tumor cells. It remains to be determined if simultaneous loss of SOCS1, SOCS3 and CIS occur in other tumor models and human cancers. While functions of the other five SOCS family members are less well characterized, we cannot exclude the possibility of functional redundancy among them. For example, hypermethylation of SOCS2 gene was reported in some ovarian and breast carcinoma cell lines in conjunction with SOCS1 or SOCS3 DNA methylation (24). Better understanding of their functions should provide additional insights into their regulation in tumorigenesis.
Another important finding from our studies is that different mechanisms contribute to the loss of SOCS1 and SOCS3 expression in Lck-transformed LSTRA cells. Epigenetic modification through DNA hypermethylation has been widely observed in SOCS gene silencing in tumor cells. Consistent with previous reports, our data also support an important role of DNA hypermethylation in SOCS1 gene silencing in LSTRA cells. On the contrary, rapid SOCS3 induction by pervanadate stimulation in LSTRA cells suggests a mechanism other than DNA hypermethylation leading to loss of SOCS3 expression. These findings provide evidence for multiple mechanisms underlying the absence of SOCS gene expression in human cancers. We showed previously a direct correlation between incomplete STAT5b modification and the lack of CIS expression in LSTRA (32). The presence of STAT5-binding sites in both $C I S$ and SOCS3 gene promoters suggests that incomplete STAT5b modification may also be involved in the loss of SOCS3 expression. Experiments are in progress to further characterize this novel mechanism and to determine whether it is shared by other SOCS family members.

Our results clearly demonstrate that exogenous SOCS1 or SOCS3 expression inhibits oncogenic Lck kinase activity and downstream STAT5b functions. We reported previously that STAT5b activation is critical in Lck-mediated oncogenesis (31). Therefore, attenuation of Lck-STAT5b signaling may contribute to the subsequent biological effects, including reduced cell proliferation and augmented apoptosis, in Lcktransformed cells. Lck is a Src-family kinase and shares high degree of structural and functional similarities with the Src kinase. However, Iwamoto et al reported that exogenous SOCS1 failed to reduce tyrosine phosphorylation of STAT3 in v-Src-transformed fibroblasts (44). It suggests that SOCS1 cannot inhibit $\mathrm{v}$-Src kinase activity, even though it was not tested directly. Enforced SOCS1 also cannot reverse v-Srcinduced cellular transformation in NIH3T3 cells (44). These discrepancies suggest that SOCS1 may interact differently with these two closely related tyrosine kinases. Alternatively, the intracellular environment of transformed cells may greatly affect SOCS1 tumor suppressing activity. It remains to be determined whether and how SOCS3 or other SOCS family members behave differently in cells transformed by the oncogenic Src kinase.

All eight SOCS family proteins share a conserved SOCS box in their carboxy-termini. The SOCS box plays a key role in ubiquitination and subsequent proteasomal degradation of SOCS proteins and some of their binding partners (45). SOCS1 accelerates TEL-JAK2 degradation through the ubiquitinproteasome pathway (46) and it contributes to SOCS1 tumorsuppressing activity in TEL-JAK2-transformed cells (47). Ectopic SOCS1 expression also leads to reduced levels of $\mathrm{v}-\mathrm{Abl}$ protein expression in co-transfected COS7 cells (8). In our studies, however, no detectable degradation of oncogenic Lck (Y505F) proteins was observed in transformed BaF3 cells transfected with SOCS1 or SOCS3 (Fig. 5). Therefore, inhibition of Lck kinase activity, but not degradation of Lck proteins, contributes mostly to the tumor suppressor functions of SOCS1 and SOCS3 in Lck-transformed BaF3 cells. We cannot exclude the possibility that proteolysis of Lck proteins may be involved in SOCS-mediated tumor suppression in a different cellular context. 
In summary, this is the first report confirming tumor suppressor activity of SOCS1 and SOCS3 toward oncogenic Lck kinase. Both SOCS1 and SOCS3 are not expressed in Lck-transformed cells, highlighting the importance of silencing both genes to create an intracellular environment favorable for STAT activation and tumor progression. Nevertheless, loss of SOCS1 and SOCS3 gene expression is mediated by different mechanisms in Lck-transformed cells. Further characterization of SOCS gene expression in other tumor cells will provide important information in understanding the roles of different SOCS family members in malignant progression of human cancers.

\section{Acknowledgements}

We thank Dr Tracy Willson (WEHI, Australia) for pEFFLAG-I/mSOCS 1 and pEF-FLAG-I/mSOCS3 expression constructs and Dr Elizabeth Yang (Vanderbilt University, Nashville, TN, USA) for Bcl-xL and GAPDH constructs. U266 cells were a kind gift from Dr Richard Jove (City of Hope Comprehensive Cancer Center, Duarte, CA, USA). This study was supported in part by National Cancer Institute grant CA107210 and the Rosalind Franklin University of Medicine and Science H.M. Bligh Cancer Research Fund (to C.L. Yu).

\section{References}

1. Croker BA, Kiu H and Nicholson SE: SOCS regulation of the JAK/STAT signalling pathway. Sem Cell Dev Biol 19: 414-422, 2008.

2. Alexander WS, Starr R, Fenner JE, Scott CL, Handman E, Sprigg NS, Corbin JE, Cornish AL, Darwiche R, Owczarek CM, Kay TW, Nicola NA, Hertzog PJ, Metcalf D and Hilton DJ: SOCS1 is a critical inhibitor of interferon gamma signaling and prevents the potentially fatal neonatal actions of this cytokine. Cell 98: 597-608, 1999.

3. Roberts AW, Robb L, Rakar S, Hartley L, Cluse L, Nicola NA, Metcalf D, Hilton DJ and Alexander WS: Placental defects and embryonic lethality in mice lacking suppressor of cytokine signaling 3. Proc Natl Acad Sci USA 98: 9324-9329, 2001.

4. Marine JC, McKay C, Wang D, Topham DJ, Parganas E, Nakajima H, Pendeville H, Yasukawa H, Sasaki A, Yoshimura A and Ihle JN: SOCS3 is essential in the regulation of fetal liver erythropoiesis. Cell 98: 617-627, 1999.

5. Liu E, Cote JF and Vuori K: Negative regulation of FAK signaling by SOCS proteins. EMBO J 22: 5036-5046, 2003.

6. Liu L, Gao Y, Qiu H, Miller WT, Poli V and Reich NC: Identification of STAT3 as a specific substrate of breast tumor kinase. Oncogene 25: 4904-4912, 2006.

7. Niwa Y, Kanda H, Shikauchi Y, Saiura A, Matsubara K, Kitagawa T, Yamamoto J, Kubo T and Yoshikawa H: Methylation silencing of SOCS-3 promotes cell growth and migration by enhancing JAK/STAT and FAK signalings in human hepatocellular carcinoma. Oncogene 24: 6406-6417, 2005.

8. Rottapel R, Ilangumaran S, Neale C, La Rose J, Ho JM, Nguyen MH, Barber D, Dubreuil P and de Sepulveda P: The tumor suppressor activity of SOCS-1. Oncogene 21: 4351-4362, 2002.

9. Limnander A, Danial NN and Rothman PB: v-Abl signaling disrupts SOCS-1 function in transformed pre-B cells. Mol Cell 15: 329-341, 2004

10. Yu H and Jove R: The STATs of cancer - new molecular target come of age. Nat Rev Cancer 4: 97-105, 2004

11. Yoshikawa H, Matsubara K, Qian GS, Jackson P, Groopman JD, Manning JE, Harris CC and Herman JG: SOCS-1, a negative regulator of the JAK/STAT pathway, is silenced by methylation in human hepatocellular carcinoma and shows growth-suppression activity. Nat Genet 28: 29-35, 2001.

12. Galm O, Yoshikawa H, Esteller M, Osieka R and Herman JG: SOCS-1, a negative regulator of cytokine signaling, is frequently silenced by methylation in multiple myeloma. Blood 101 : 2784-2788, 2003.
13. Liu TC, Lin SF, Chang JG, Yang MY, Hung SY and Chang CS: Epigenetic alteration of the SOCS1 gene in chronic myeloid leukaemia. Br J Haematol 123: 654-661, 2003.

14. Watanabe D, Ezoe S, Fujimoto M, Kimura A, Saito Y, Nagai H, Tachibana I, Matsumura I, Tanaka T, Kanegane H, Miyawaki T, Emi M, Kanakura Y, Kawase I, Naka T and Kishimoto T: Suppressor of cytokine signalling-1 gene silencing in acute myeloid leukaemia and human haematopoietic cell lines. Br J Haematol 126: 726-735, 2004.

15. Fukushima N, Sato N, Sahin F, Su GH, Hruban RH and Goggins M: Aberrant methylation of suppressor of cytokine signalling-1 (SOCS-1) gene in pancreatic ductal neoplasms. Br J Cancer 89: 338-343, 2003

16. Nagai H, Naka T, Terada Y, Komazaki T, Yabe A, Jin E, Kawanami O, Kishimoto T, Konishi N, Nakamura M, Kobayashi Y and Emi M: Hypermethylation associated with inactivation of the SOCS-1 gene, a JAK/STAT inhibitor, in human hepatoblastomas. J Hum Genet 48: 65-69, 2003.

17. Fujitake S, Hibi K, Okochi O, Kodera Y, Ito K, Akiyama S and Nakao A: Aberrant methylation of SOCS-1 was observed in younger colorectal cancer patients. J Gastroenterol 39: 120-124, 2004 .

18. Oshimo Y, Kuraoka K, Nakayama H, Kitadai Y, Yoshida K, Chayama $\mathrm{K}$ and Yasui W: Epigenetic inactivation of SOCS-1 by $\mathrm{CpG}$ island hypermethylation in human gastric carcinoma. Int J Cancer 112: 1003-1009, 2004.

19. To KF, Chan MW, Leung WK, Ng EK, Yu J, Bai AH, Lo AW, Chu SH, Tong JH, Lo KW, Sung JJ and Chan FK: Constitutional activation of IL-6-mediated JAK/STAT pathway through hypermethylation of SOCS-1 in human gastric cancer cell line. Br J Cancer 91: 1335-1341, 2004.

20. Isomoto H, Mott JL, Kobayashi S, Werneburg NW, Bronk SF, Haan S and Gores GJ: Sustained IL-6/STAT-3 signaling in cholangiocarcinoma cells due to SOCS-3 epigenetic silencing. Gastroenterology 132: 384-396, 2007.

21. Weber A, Hengge UR, Bardenheuer W, Tischoff I, Sommerer F, Markwarth A, Dietz A, Wittekind C and Tannapfel A: SOCS-3 is frequently methylated in head and neck squamous cell carcinoma and its precursor lesions and causes growth inhibition. Oncogene 24: 6699-6708, 2005.

22. He B, You L, Uematsu K, Zang K, Xu Z, Lee AY, Costello JF, McCormick F and Jablons DM: SOCS-3 is frequently silenced by hypermethylation and suppresses cell growth in human lung cancer. Proc Natl Acad Sci USA 100: 14133-14138, 2003.

23. Tischoff I, Hengge UR, Vieth M, Ell C, Stolte M, Weber A, Schmidt WE and Tannapfel A: Methylation of SOCS-3 and SOCS-1 in the carcinogenesis of Barrett's adenocarcinoma. Gut 56: 1047-1053, 2007.

24. Sutherland KD, Lindeman GJ, Choong DY, Wittlin S, Brentzell L, Phillips W, Campbell IG and Visvader JE: Differential hypermethylation of SOCS genes in ovarian and breast carcinomas. Oncogene 23: 7726-7733, 2004.

25. Kim LC, Song L and Haura EB: Src kinases as therapeutic targets for cancer. Nat Rev Clin Oncol 6: 587-595, 2009.

26. Sefton BM: The lck tyrosine protein kinase. Oncogene 6: 683-686, 1991.

27. Abraham KM, Levin SD, Marth JD, Forbush KA and Perlmutter RM: Thymic tumorigenesis induced by overexpression of p56lck. Proc Natl Acad Sci USA 88: 3977-3981, 1991.

28. Majolini MB, Boncristiano M and Baldari CT: Dysregulation of the protein tyrosine kinase LCK in lymphoproliferative disorders and in other neoplasias. Leuk Lymphoma 35: 245-254, 1999.

29. Wright DD, Sefton BM and Kamps MP: Oncogenic activation of the Lck protein accompanies translocation of the LCK gene in the human HSB2 T-cell leukemia. Mol Cell Biol 14: 2429-2437, 1994.

30. Yu CL, Jove R and Burakoff SJ: Constitutive activation of the Janus kinase-STAT pathway in T lymphoma overexpressing the Lck protein tyrosine kinase. J Immunol 159: 5206-5210, 1997.

31. Shi M, Cooper JC and Yu CL: A constitutively active Lck kinase promotes cell proliferation and resistance to apoptosis through signal transducer and activator of transcription $5 \mathrm{~b}$ activation. Mol Cancer Res 4: 39-45, 2006.

32. Cooper JC, Boustead JN and Yu CL: Characterization of STAT5B phosphorylation correlating with expression of cytokine-inducible SH2-containing protein (CIS). Cell Signal 18: 851-860, 2006.

33. Yu CL, Prochownik EV, Imperiale MJ and Jove R: Attenuation of serum inducibility of immediate early genes by oncoproteins in tyrosine kinase signaling pathways. Mol Cell Biol 13: 2011-2019, 1993 
34. Ammanamanchi S and Brattain MG: 5-azaC treatment enhances expression of transforming growth factor-beta receptors through down-regulation of Sp3. J Biol Chem 276: 32854-32859, 2001.

35. Marth JD, Peet R, Krebs EG and Perlmutter RM: A lymphocytespecific protein-tyrosine kinase gene is rearranged and overexpressed in the murine T cell lymphoma LSTRA. Cell 43: 393-404, 1985.

36. Socolovsky M, Fallon AEJ, Wang S, Brugnara C and Lodish HF Fetal anemia and apoptosis of red cell progenitors in Stat5a $\mathrm{a}^{-/-5 \mathrm{~b}^{-/}}$ mice: a direct role for STAT5 in Bcl-XL induction. Cell 98: 181-191, 1999.

37. Ruff SJ, Chen K and Cohen S: Peroxovanadate induces tyrosine phosphorylation of multiple signaling proteins in mouse liver and kidney. J Biol Chem 272: 1263-1267, 1997.

38. Goodman JI and Watson RE: Altered DNA methylation: a secondary mechanism involved in carcinogenesis. Annu Rev Pharmacol Toxicol 42: 501-525, 2002.

39. Boggon TJ and Eck MJ: Structure and regulation of Src family kinases. Oncogene 23: 7918-7927, 2004

40. Rodriguez-Tarduchy G, Collins M and Lopez-Rivas A: Regulation of apoptosis in interleukin-3-dependent hemopoietic cells by interleukin-3 and calcium ionophores. EMBO J 9: 2997-3002, 1990.

41. Van Engeland M, Nieland LJ, Ramaekers FC, Schutte B and Reutelingsperger CP: Annexin V-affinity assay: a review on an apoptosis detection system based on phosphatidylserine exposure. Cytometry 31: 1-9, 1998.
42. Wyllie AH: Glucocorticoid-induced thymocyte apoptosis is associated with endogenous endonuclease activation. Nature 284: 555-556, 1980.

43. Darnell JE Jr: STATs and gene regulation. Science 277: 1630-1635, 1997.

44. Iwamoto T, Senga T, Naito Y, Matsuda S, Miyake Y, Yoshimura A and Hamaguchi M: The JAK-inhibitor, JAB/SOCS-1 selectively inhibits cytokine-induced, but not v-Src induced JAK-STAT activation. Oncogene 19: 4795-4801, 2000

45. Kile BT, Schulman BA, Alexander WS, Nicola NA, Martin HME and Hilton DJ: The SOCS box: a tale of destruction and degradation. Trends Biochem Sci 27: 235-241, 2002.

46. Kamizono S, Hanada T, Yasukawa H, Minoguchi S, Kato R, Minoguchi M, Hattori K, Hatakeyama S, Yada M, Morita S, Kitamura T, Kato H, Nakayama $\mathrm{K}$ and Yoshimura A: The SOCS box of SOCS-1 accelerates ubiquitin-dependent proteolysis of TEL-JAK2. J Biol Chem 276: 12530-12538, 2001.

47. Frantsve J, Schwaller J, Sternberg DW, Kutok J and Gilliland DG: Socs-1 inhibits TEL-JAK2-mediated transformation of hematopoietic cells through inhibition of JAK2 kinase activity and induction of proteasome-mediated degradation. Mol Cell Biol 21: 3547-3557, 2001 . 